\title{
Procaine and procainamide inhibit the Wnt canonical pathway by promoter demethylation of WIF-1 in lung cancer cells
}

\author{
ZHI GAO ${ }^{1,3}$, ZHIDONG XU ${ }^{3}$, MING-SZU HUNG ${ }^{3,4}$, YU-CHING LIN ${ }^{4}$, TIANYOU WANG $^{1}$, \\ MIN GONG $^{1}$, XIUYI ZHI ${ }^{2}$, DAVID M. JABLONS ${ }^{3}$ and LIANG YOU ${ }^{3}$ \\ ${ }^{1}$ Department of Cardiothoracic Surgery, Beijing Friendship Hospital and ${ }^{2}$ Beijing Xuanwu Hospital, Capital Medical \\ University, Beijing, P.R. China; ${ }^{3}$ Thoracic Oncology Laboratory, Department of Surgery, UCSF Comprehensive \\ Cancer Center, University of California, San Francisco, CA 94143-0128, USA; ${ }^{4}$ Department of Internal Medicine, \\ Division of Pulmonary and Critical Care Medicine, Chang Gung Memorial Hospital, Chiayi, Taiwan, R.O.C.
}

Received June 10, 2009; Accepted July 30, 2009

DOI: 10.3892/or_00000590

\begin{abstract}
Secreted Wingless type (Wnt) ligands have previously been shown to be involved in tumor developmental processes and oncogenesis. Aberrant promoter methylation of Wnt inhibitory factor-1 (WIF-1) is a fundamental mechanism of epigenetic silencing in human cancers. Procaine, a local anesthetic drug, and procainamide, a drug for the treatment of cardiac arrhythmias, have been reported as inhibitors of DNA methylation, causing demethylation and reactivation of methylation-silenced genes such as RARß and GSTP1. The promoter demethylation of $W I F-1$ has not previously been reported on. We demonstrated previously that WIF-1 is silenced due to promoter hypermethylation in lung cancer cell lines. In this study, we demonstrate promoter demethylation of $W I F-1$; restoration of WIF-1 expression, and underexpression of cytosolic $\beta$-catenin protein and TCF reporter activity, after procaine and procainamide treatment in H460 and A549 cell lines. Our results provide the first evidence that procaine and procainamide reactivate $W I F-1$ in these cancer cells and downregulate the Wnt canonical pathway. These results further suggest that procaine and procainamide may have a potential use for preventing the development of lung cancer.
\end{abstract}

\section{Introduction}

Silencing of the promoter methylation $\mathrm{X}$ of tumor suppressor genes is recognized as being a molecular hallmark of human

Correspondence to: Dr D.M. Jablons and Dr L. You, Department of Surgery, University of California, San Francisco, Box 0128, San Francisco, CA 94143-0128, USA

E-mail: david.jablons@ucsfmedctr.org

E-mail: liang.you@ucsfmedctr.org

Key words: Wnt inhibitory factor-1, promoter hypermethylation, demethylation, procaine, procainamide cancer (1-4). Nearly $50 \%$ of all proven tumor suppressor genes can be silenced by hypermethylation along with many genes that play putative roles in antitumor activities (5).

The Wingless-type (Wnt) family of secreted glycoproteins is a group of signaling molecules that have been implicated in oncogenesis $(6,7)$. The proto-oncogenic effects of WNT were discovered more than 20 years ago (8). Since then, there have been numerous demonstrations of aberrant Wnt signaling pathway activation in disparate human cancers including colorectal cancer (9), head and neck carcinoma (10), melanoma (11), and leukemia (12). We previously reported overexpression of disheveled (Dvl) and $B$-catenin proteins in mesothelioma, nasopharyngeal carcinoma (NPC) and nonsmall cell lung cancer (NSCLC) (13-15).

Wnt inhibitory factor-1 (WIF-1) is a Wnt antagonist that inhibits Wnt signaling by direct binding to Wnt molecules. WIF-1 silencing, due to promoter hypermethylation, has been observed in NSCLC, NPC, gastrointestinal malignancies, melanoma and Barrett's esophagus (16-18). Previously, we cloned the WIF-1 promoter in NSCLC and colorectal cancer cell lines (19).

Procaine, a drug approved by the FDA for use as a local anesthetic and procainamide, FDA approved for treatment of cardiac arrhythmias, are derivatives of 4-aminobenzoic acid. However, procaine is the ester with 2-(diethylamino)ethanol while procainamide is the amide with 2-(diethylamino) ethylamine. Procaine was previously reported to be an inhibitor of DNA methylation in breast cancer cells, causing demethylation and reactivation of tumor suppressor genes with hypermethylated $\mathrm{CpG}$ islands (20). Procainamide has been indicated as an inhibitor of DNA methylation, specifically causing DNA demethylation (21) and restoring expression of the GSTP1 gene in prostate cancer cells (22), and expression of $E R, R A R \beta$, and $p 16$ genes in bladder and breast cancer cells (23), where previously silenced by hypermethylation. However, the promoter demethylation of WIF-1 has not previously been reported. Herein, we hypothesize that procaine and procainamide may be used as an effective and nontoxic demethylating agents of the WIF-1 promoter. 
A

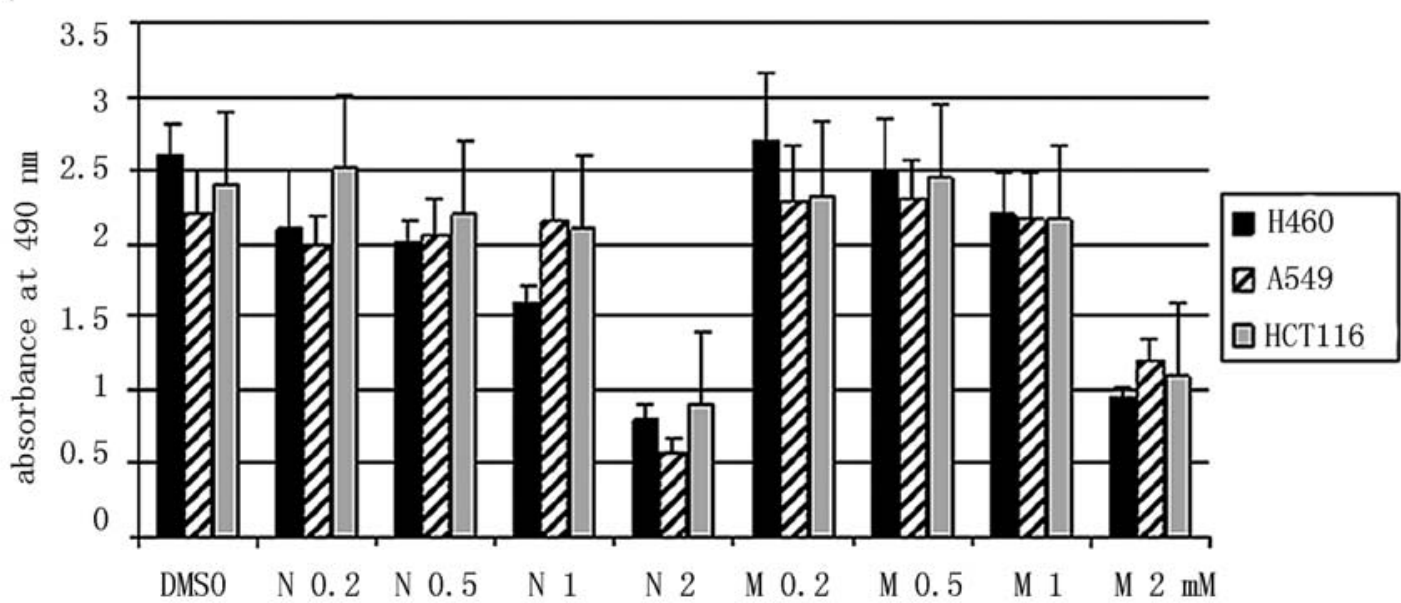

B H460 A549 Normal Lung H1703 HCT116

WIF-1

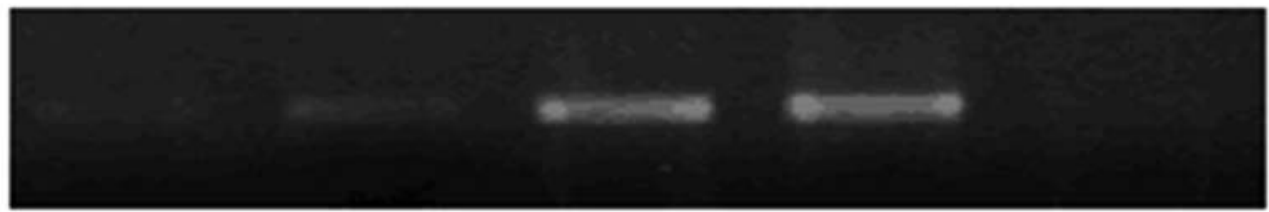

GAPDH

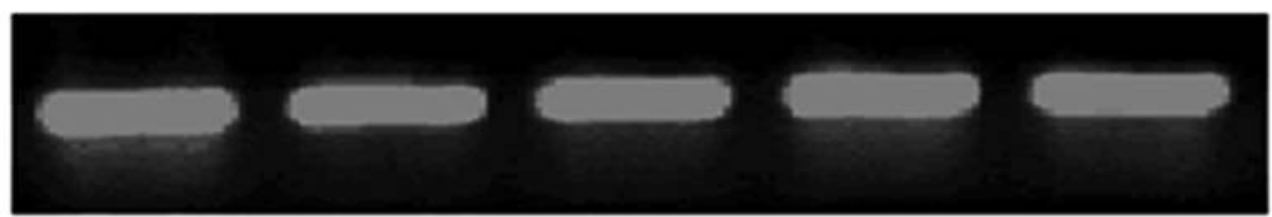

Figure 1. Proliferation effects of procaine and procainamide in H460, A549 and HCT116 cells (A). Cells were treated with procaine and procainamine (0-2 mM) and incubated for $72 \mathrm{~h}$ and proliferation was assessed by MTS assay. Experiments were performed independently at least 3 times; control carrier DMSO $(0 \mathrm{mM})$. Data are the means $\pm \mathrm{SD}, \mathrm{N}$ and $\mathrm{M}$ represent procaine and procainamine, respectively. (B) Reverse transcriptase-PCR for WIF-1. The amplified human cDNA fragment is $451 \mathrm{bp}$. The H1703 and HCT116 were included as positive and negative control, respectively. Glyceraldehyde-3-phosphate dehydrogenase $(G A P D H)$ was used as a control for RNA quality and loading.

\section{Materials and methods}

Cell lines, tissue samples and chemical treatment. NSCLC cell lines (H1703, H460, and A549) and colorectal cancer cell line HCT116 were obtained from American Type Culture Collections (Manassas, VA). Cells were cultured in RPMI1640 supplemented with $10 \%$ fetal bovine serum, penicillin (100 IU/ml), and streptomycin $(100 \mu \mathrm{g} / \mathrm{ml})$. All cells were cultured at $37^{\circ} \mathrm{C}$ in a humid incubator with $5 \% \mathrm{CO}_{2}$. Normal lung tissues from patients undergoing resection for lung cancers were collected at the time of surgery and immediately snap-frozen in liquid nitrogen (Institutional Review Board approval H8714(-15319-040). These tissue samples were kept at $-170^{\circ} \mathrm{C}$ in a liquid nitrogen before use. Procaine, procainamide and 5-Aza-2'-deoxycytidine (DAC) was obtained from Sigma-Aldrich (St. Louis, MO, USA). They were prepared as stock solutions in dimethyl sulfoxide (DMSO), diluted in media and sterile filtered before use. To restore WIF- 1 expression, $1 \times 10^{5}$ cells were seeded into 6-well culture plates. After 24 -h culture, cells were treated with procaine, procainamide $(0-1 \mathrm{mM})$ and DAC $(20 \mu \mathrm{M})$ for $72 \mathrm{~h}$, treatment and harvested as mentioned previously (24).
Cell proliferation. H460, A549, and HCT116 cells were plated in 96-well plates at a density of 5000 cells/well. Cells were allowed to attach overnight in growth medium. After $24 \mathrm{~h}$, H460, A549, and HCT116 cells were treated with procaine and procainamide (0-2 mM). After incubation for $72 \mathrm{~h}$, cellular proliferation was measured using the MTS assay and absorbance was measured at $490 \mathrm{~nm}$. Proliferation data are presented as means $\pm \mathrm{SD}$.

Semiquantitative reverse transcription-PCR. Total RNA from H460, A549, HCT116, and H1703 cell lines and normal lung tissue was isolated using an extraction kit (RNeasy mini kit; Qiagen, Valencia, CA, USA). Reverse transcription-PCR (RT-PCR) was performed in Bio-RAD iCycler (Bio-RAD Laboratories, Hercules, CA, USA) using an RT-PCR kit (SuperScript One-step RT-PCR with Platinum Taq kit; Invitrogen, Carlsbad, CA, USA) according to the manufacturer's protocol. Primer sequences for the human WIF-1 cDNA were 5'-CCGAAATGGAGGCTTTTGTA-3' (forward) and 5'-GTGTCTTCCATGCCAACCTT-3' (reverse). Glyceraldehyde-3-phosphatedehydrogenase (GAPDH) was used as an internal control. 



Figure 2. Methylation and pharmacologic demethylation of WIF-1 promotor region with procaine $(\mathrm{N})$, procainamine $(\mathrm{M})$ and DAC treatment in $\mathrm{H} 460$ and A549 cells. (A) Methylation-specific PCR analysis of the $\mathrm{CpG}$ island of cell lines, whereas treatment with procaine and procainamide $(0-1 \mathrm{mM})$, DAC was used as a positive demethylation control. Bands in lane $\mathrm{U}$ are unmethylated DNA product with unmethylation-specific primers. Bands in lane $\mathrm{M}$ are methylated DNA product amplified with methylation-specific primers. (B) Bisulfite genomic DNA sequencing analysis. $\square$ and $\boldsymbol{\square}$ represent unmethylated and methylated $\mathrm{CpG}$ islands, respectively; percentage indicate the fraction of methylated $\mathrm{CpG}$ islands. We sequenced 3 clones of PCR products amplified from bisulfite-treated genomic DNA for each cell line.

Methylation-specific PCR (MSP). Genomic DNA from the cell lines and normal lung tissue was extracted using DNeasy blood and tissue kit (Qiagen), according to the manufacturer's protocol. Bisulfite modification of genomic DNA was performed by using a methylation kit (EZDNA methylation kit; Zymo Research, Orange, CA, USA). Bisulfite-treated genomic DNA was amplified using either a methylationspecific or an unmethylation-specific primer set. HotStarTaq DNA polymerase (Qiagen) was used in the experiments. Sequences of the methylation-specific primers were $5^{\prime}-\mathrm{TCG}$ CGGGCGTTTTATTGGGC-3' (forward) and 5'-AACGA AACCAACAATCAACG -3' (reverse). Sequences of the unmethylation-specific primers were 5'-TTGTGGGTGT TTTATTGGGT-3' (forward) and 5'-AACAAAACCAAC AATCAACA-3' (reverse).

Sequencing analysis. Bisulfite-treated genomic DNA was amplified using two pairs of primers: 5'-GAGTGATGTTTT
AGGGGTTT-3' (forward) and 5'-CCTAAATACCAAAAAA CCTAC-3' (reverse), designed to amplify nucleotides -554 to -140 of the WIF- 1 promoter region; and 5'-GTAGGTTTTT TGGTATTTAGG-3' (forward) and 5'-TCCATAAATACA AACTCTCCTC-3' (reverse), to amplify nucleotides -161 to +118 (the start codon ATG of WIF-1 is defined as +1 ). The PCR products were extracted from the agarose gel using an extraction kit (QIAquick gel extraction kit; Qiagen) and were subsequently sequenced at MCLab (South San Francisco, CA, USA).

Transient transfection and luciferase reporter assay. The TOP/ FOP flash plasmid system was used to determine $\beta$-catenininduced Tcf/Lef transcriptional activity (25). All transfection experiments were performed using the Lipofectamine 2000 method (Invitrogen) in triplicate in accordance with the manufacturer's instructions. The HCT116 cells were transfected with $8 \mu \mathrm{g}$ Super8X TOP-flash or $8 \mu \mathrm{g}$ Super8X FOP (the mutated control) flash plasmid (as a kind gift from Professor R.T. Moon), the pRL-TK plasmid (Promega, Madison, WI) was co-transfected to normalize for transfection efficiency. Cells were treated with procaine and procainamide $(0-1 \mathrm{mM})$ $24 \mathrm{~h}$ after transfection Luciferase activity was assayed $24 \mathrm{~h}$ after treatment by using the Dual-Luciferase reporter assay system (Promega).

Western blotting. Cytosolic proteins were prepared as previously described (26). The proteins were separated on 4-15\% gradient SDS-polyacrylamide gels and transferred to Immobilon-P membranes (Millipore, Bellerica, MA, USA). The proteins were bound with the primary antibodies of ß-catenin (Transduction Laboratories, Lexington, KY, USA) and $B$-actin (Sigma Chemical). Antigen-antibody complexes were detected by ECL blotting analysis system (Amersham Pharmacia Biotech, Piscataway, NJ, USA).

\section{Results}

Effect of procaine and procainamide on cell proliferation. We analyzed the effects of procaine and procainamide on cell proliferation in H460, A549, and HCT116 cell line using MTS assay (Fig. 1A). We compared with that of controls, the remaining cell viability was not altered at a concentration $\leq 1 \mathrm{mM}$ and reduced at concentrations of $2 \mathrm{mM}$ Therefore, the treatment of procaine and procainamide, at the concentrations ranging from 0 to $1 \mathrm{mM}$, has no cytotoxic effects on the H460, A549, and HCT116 cells during 72-h incubation period.

Silencing and promoter methylation of WIF-1 in H460 and A549 cell lines. The methods to identify the WIF-1 promoter have been described previously (16), We confirm that the 5 ' region of the gene presents classical features of a promoter region and map $106 \mathrm{CpGs}$ islands within the WIF-1 promoter. $W I F-1$ expression was examined as previous described $(16,27)$. We found that the WIF- 1 transcript was missing in $\mathrm{H} 460$, A549 and HCT116 cell lines. In contrast, it was expressed in H1703 cells and normal lung (Fig. 1B). Promoter methylation was found by using methylation-specific PCR (MSP) in H460 and A549 cell lines (Fig. 2A). Consistent with MSP results, 



Figure 3. Reactivation of WIF-1 expression in cell lines. Reverse transcriptionPCR was performed after procaine and procainamide (0-1 $\mathrm{mM})$ and DAC $(20 \mu \mathrm{M})$ treatment for $72 \mathrm{~h}$.

we found that these $\mathrm{CpG}$ islands in $\mathrm{H} 460$ and A549 cell lines were densely methylated by using bisulfite genomic DNA sequencing technique. In contrast, $\mathrm{CpG}$ islands in normal lung tissue were unmethylated (Fig. 2B).

Promoter demethylation of WIF-1 gene with procaine and procainamide treatment. We analyzed the methylation status of CpG islands in the WIF-1 promoter region in H460 and A549 cell lines after incubation with procaine and procainamide (0-1 mM) for 3 days. By using MSP technique, the WIF-1 promoter region revealed concentration-dependent demethylation (Fig. 2A), DAC was used as a positive demethylation control (15). We also used bisulfite genomic DNA sequencing to analyze details of the methylation status of $64 \mathrm{CpG}$ sites in the $672 \mathrm{bp}$ of the WIF-1 promoter region. The sequencing results showed that the $W I F-1$ promoter methylation level decreased from $77.6 \%$ to $25.5 \%$ and $36.9 \%$ after treatment with $0.5 \mathrm{mM}$ procaine and procainamide in $\mathrm{H} 460$ cell line and from $76.5 \%$ to $23.9 \%$ and $33.3 \%$ in A549 cell line (Fig. 2B).

Restoration of WIF-1 expression with procaine and procainamide treatment. Then we analyzed the expression level of WIF-1 by semiquantitative RT-PCR. In agreement with the MSP and sequencing data, the results revealed detectable and concentration-dependent reactivation after treatment by procaine for 3 days, but no concentration-dependent reactivation after treatment by procainamide (Fig. 3). These results suggest that WIF-1 silence in $\mathrm{H} 460$ and A549 cell lines correlates with dense methylation of WIF-1 promoter region and can be restored by procaine and procainamide.

Downregulation of the Wnt canonical pathway. Downregulation of the Wnt canonical pathway was confirmed of Tcf/Lef transcriptional activity and cytosolic $\beta$-catenin protein level. The TOP/FOP flash plasmid system was used to determine Tcf/Lef activity, the results revealed concentrationdependent decrease of TOP/FOP luciferase activity after

TOP/FOP

A

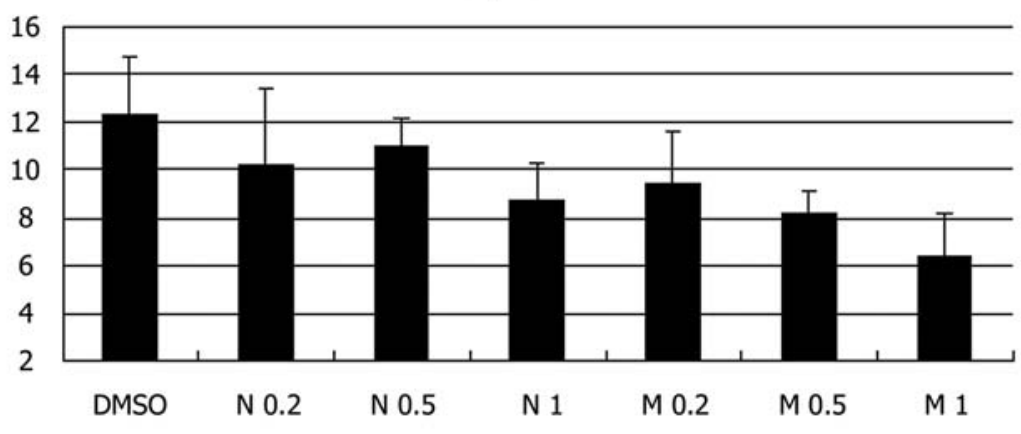

B



A549


Figure 4. TOP/FOP measurement by using the Dual-Luciferase reporter assay system (A). Cells were treated with procaine and procainamide (0-1 mM). Data are the means $\pm \mathrm{SD}$. (B) Expression of $ß$-catenin proteins after procaine and procainamide $(0.5 \mathrm{mM})$ treatment for 3 days in $\mathrm{H} 460$ and $\mathrm{A} 549$ cells; protein levels were determined with Western blot analysis using ß-actin as an internal control. 
treatment by procaine and procainamide (Fig. 4A). We subsequently measured expression of B-catenin by Western blot analysis using $\beta$-actin as an internal control, and found that $\mathrm{H} 460$ and A549 cells had slightly decreased expression of $B$-catenin after treatment by procaine and dramatically decreased expression by procainamide (Fig. 4B).

\section{Discussion}

Wnt signaling has emerged as a critical pathway in lung carcinogenesis. This has been amply demonstrated in numerous cancers $(28,29)$. Several Wnt pathway proteins, including Wnt-1, Dvl-3 and B-catenin, have also been shown to be overexpressed in a number of cancers.

Wnt inhibitory factor-1 (WIF-1), a highly conserved gene that was first identified in the human retina, is a Wnt antagonist that inhibits Wnt signaling by direct binding to Wnt molecules. WIF-1 downregulates the Wnt pathway and inhibits NSCLC cell growth. Transfection with WIF-1 induces apoptosis in NSCLC cells and inhibits colony formation. Transfection by injecting WIF-1 plasmid also inhibits NSCLC tumor xenograft growth (16).

A growing list of genes have been identified that evidence abnormal CpG island promoter methylation (30). Methylation of the $\mathrm{CpG}$ island within the functional promoter region of WIF- 1 is an important mechanism of aberrant Wnt signaling activation in cancer. Thus, by using methylation specific PCR and sequence analysis after bisulfite treatment, we demonstrated the frequent hypermethylation of the $\mathrm{CpG}$ islands in the functional WIF-1 promoter region, the hypermethylation correlated with their transcriptional silencing in human lung cancer cell lines (16). Conversely, promoter demethylation of $W I F-1$ restored the silenced WIF-1.

Procaine has been previously reported, in breast cancer cells, to cause global DNA hypomethylation, growth inhibition, and demethylation and re-expression of a methylation-silenced RARß2 (20). As such, procaine behaves very similarly to procainamide, the latter which specifically inhibits the hemimethylase activity of DNA methyltransferase 1 (DNMT1) (31), restores the expression of the hypermethylated GSTPI gene in prostate cancer cells, and diminishes xenograft tumor growth (23). Therefore, we hypothesize that procaine and procainamide can also reactivate methylation-silenced WIF-1 gene.

In this study, we demonstrated that untreated H460 and A549 cells which were methylated silenced the expression of the WIF-1 gene. However, after exposure to procaine and procainamide, this epigenetic change was reversed. We also showed that $\mathrm{H} 460$ and A549 cells treated with procaine and procainamide decreased Tcf/Lef activity and expression of Bcatenin using a TOP/FOP Dual-Luciferase reporter assay and Western blot analysis, respectively.

These results are consistent with our hypothesis that procaine and procainamide may, in addition to restoring the methylated WIF-1 by demethylation, downregulate the Wnt canonical pathway in $\mathrm{H} 460$ and A549 cell lines.

The use of the nucleoside analogs such as DAC in clinical trials has been limited due to their side effects, including thrombocytopenia and neutropenia, probably caused by cytotoxic effects associated with the incorporation of the drugs into DNA, apart from their DNA-hypomethylation value. There is thus an urgent need to discover less toxic demethylating agents that are not incorporated into DNA. Procaine and procainamide have been proposed as non-nucleoside inhibitors of DNA methylation. Their side effects are expected to be less as seen in nucleoside DNA methylation inhibitors because of their widespread clinical use (32).

We provided the first evidence that procaine and procainamide reactivate $W I F-1$ from a previously silenced methylation state and downregulate the canonical Wnt pathway. Procaine and procainmide may have a potential use for preventing the development of cancer.

\section{Acknowledgements}

This work was supported by NIH grant RO1 CA 093708-01A3, and the Larry Hall memorial trust and Bonnie J. and Anthony Addario; the Bonnie J. Addario Lung Cancer Foundation; the Eileen D. Ludwig Endowed for Thoracic Oncology Research, Kazan, McClain, Abrams, Fernandez, Lyons, Greenwood, Harley \& Oberman Foundation, Inc.; Estate of Robert Griffiths; Estate of Norman Mancini; the Barbara Isackson Lung Cancer Research Fund. We thank Mr. Richard N. Barg, JD/MBA of the Thoracic Oncology Program and the Department of Surgery at the University of California, San Francisco for his editorial help in the preparation of this manuscript.

\section{References}

1. Herman JG and Baylin SB: Gene silencing in cancer in association with promoter hypermethylation. N Engl J Med 349: 2042-2054, 2003

2. Antequera $\mathrm{F}$ and Bird $\mathrm{A}$ : Number of $\mathrm{CpG}$ islands and genes in human and mouse. Proc Natl Acad Sci USA 90: 11995-11999, 1993.

3. Rountree MR, Bachman KE and Herman JG: DNA methylation, chromatin inheritance, and cancer. Oncogene 20: 3156-3165, 2001.

4. Jones PA and Laird PW: Cancer epigenetics comes of age. Nat Genet 21: 163-167, 1999.

5. Baylin SB: Reversal of gene silencing as a therapeutic target for cancer - roles for DNA methylation and its interdigitation with chromatin. Novartis Found Symp 259: 226-233, 2004.

6. Polakis P: Wnt signaling and cancer. Genes Dev 14: 1837-1851, 2000.

7. Lustig B and Behrens J: The Wnt signaling pathway and its role in tumor development. J Cancer Res Clin Oncol 129: 199-221, 2003.

8. Nusse R and Varmus HE: Many tumors induced by the mouse mammary tumor virus contain a provirus integrated in the same region of the host genome. Cell 31: 99-109, 1982.

9. Morin PJ, Sparks AB, Korinek V, et al: Activation of B-cateninTcf signaling in colon cancer by mutations in $\beta$-catenin or APC. Science 275: 1787-1790, 1997.

10. Rhee CS, Sen M, Lu D, et al: Wnt and frizzled receptors as potential targets for immunotherapy in head and neck squamous cell carcinomas. Oncogene 21: 6598-6605, 2002.

11. Weeraratna AT, Jiang Y, Hostetter G, et al: Wnt5a signaling directly affects cell motility and invasion of metastatic melanoma. Cancer Cell 1: 279-288, 2002.

12. Lu D, Zhao Y, Tawatao R, et al: Activation of the Wnt signaling pathway in chronic lymphocytic leukemia. Proc Natl Acad Sci USA 101: 3118-3123, 2004.

13. Lin YC, You L, Xu Z, et al: Wnt inhibitory factor-1 gene transfer inhibits melanoma cell growth. Hum Gene Ther 18: 379-386, 2007.

14. Uematsu K, He B, You L, et al: Activation of the Wnt pathway in non small cell lung cancer: evidence of dishevelled overexpression. Oncogene 22: 7218-7221, 2003. 
15. Lin YC, You L and Xu ZD: Wnt signaling activation and WIF-1 silencing in nasopharyngeal cancer cell lines. Biochem Biophys Res Commun 341: 635-640, 2006.

16. Mazieres J, He B,You L, et al: Wnt inhibitory factor-1 is silenced by promoter hypermethylation in human lung cancer. Cancer Res 64: 4717-4720, 2004.

17. Yang TM, Leu SW, Li JM, et al: WIF-1 promoter region hypermethylation as an adjuvant diagnostic marker for non-small cell lung cancer-related malignant pleural effusions. J Cancer Res Clin Oncol 135: 919-924, 2009

18. Clément G, Guilleret I, He B, Yagui-Beltrán A, et al: Epigenetic alteration of the Wnt inhibitory factor-1 promoter occurs early in the carcinogenesis of Barrett's esophagus. Cancer Sci 99: 46$53,2008$.

19. Reguart B, He B, Xu ZD, et al: Cloning and characterization of the promoter of human Wnt inhibitory factor-1. Biochem Biophys Res Commun 323: 229-234, 2004.

20. Ana VG, Mario FF, Jesus E and Manel E: Procaine is a DNAdemethylating agent with growth-inhibitory effects in human cancer cells. Cancer Res 63: 4984-4989, 2003.

21. Scheinbart LS, Johnson MA, Gross LA, et al: Procainamide inhibits DNA methyltransferase in a human $\mathrm{T}$ cell line. J Rheumatol 18: 530-534, 1991.

22. Lin X, Asgari K, Putzi MJ, et al: Reversal of GSTP1 CpG island hypermethylation and reactivation of pi-class glutathione S-transferase (GSTP1) expression in human prostate cancer cells by treatment with procainamide. Cancer Res 61: 8611-8616, 2001.

23. Blanca SP, Catalina TB, Enrique PC, et al: Reactivation of tumor suppressor genes by the cardiovascular drugs hydralazine and procainamide and their potential use in cancer therapy. Clin Cancer Res 9: 1596-1603, 2003.
24. Cheung HW, Ching YP, Nicholls JM, et al: Epigenetic inactivation of CHFR in nasopharyngeal carcinoma through promoter methylation. Mol Carcinogen 43: 237-245, 2005.

25. Korinek V, Barker N, Morin PJ, et al: Constitutive transcriptional activation by a beta-catenin-Tcf complex in APC colon carcinoma. Science 275: 1784-1787, 1997.

26. Uematsu K, Kanazawa S, You L, et al: Wnt pathway activation in mesothelioma: evidence of Dishevelled overexpression and transcriptional activity of beta-catenin. Cancer Res 63: 4547-4551, 2003.

27. He B, Reguart N, You L, et al: Blockade of Wnt-1 signaling induces apoptosis in human colorectal cancer cells containing downstream mutations. Oncogene 24: 3054-3058, 2005.

28. Miller JR: The Wnts. Genome Biol 3: 1-15, 2002.

29. Van GM, Daemen MJ, Smits JF, et al: The Wnt-frizzled cascade in cardiovascular disease. Cardiovasc Res 55: 16-24, 2002.

30. Herman JG and Baylin SB: Gene silencing in cancer in association with promoter hypermethylation. N Engl J Med 349: 2042-2054, 2003.

31. Lee BH, Yegnasubramanian S, Lin X, et al: Procainamide is a specific inhibitor of DNA methyltransferase1. J Biol Chem 280: 40749-40756, 2005.

32. Cornacchia E, Golbus J, Maybaum J, et al: Hydralazine and procainamide inhibit T cell DNA methylation and induce autoreactivity. J Immunol 140: 2197-2200, 1988. 